\title{
I-V CHARACTERISTICS AND TRANSCONDUCTANCE MODELING FOR DUAL CHANNEL AIGaN/GaN MODFETs
}

\author{
Rahis Kumar Yadav ${ }^{1}$, Pankaj Pathak ${ }^{2}$, R M Mehra ${ }^{3}$ \\ ${ }^{l}$ Department of Electronics and Communication Engineering, School of Engineering and Technology, Sharda \\ University, Gr. Noida, India \\ ${ }^{2}$ Department of Electronics and Communication Engineering, School of Engineering and Technology, Sharda \\ University, Gr. Noida, India \\ ${ }^{3}$ Department of Electronics and Communication Engineering, School of Engineering and Technology, Sharda \\ University, Gr. Noida, India
}

\begin{abstract}
In this paper we present 2-D analytical model for I-V characteristics and transconductance of dual channel AlGaN/GaN Modulation Doped Field Effect Transistor (DC-MODFET) to demonstrate the current-voltage as well as transfer characteristics of the device structure under different bias conditions. The model analyses sheet charge density and finaly current density in each of the top and bottom channels using effective device threshold expressions based on spontaneous and piezoelectric polarization dependent two dimensional electron gas (2-DEG). An aluminum mole fraction graded doping profile in bottom barrier layer has been used for bottom channel sheet carrier density. Trapping/detrapping surface states are also considered to determine device drive current and trans-conductance expressions. The presence of double channels in the device minimize the current collapse problems, is accurately explained in our proposed model. The current collapse that occurs more in the top channel nearer to the gate, is subsidized by the current in bottom channel, hence minimizing overall current collapse in the device. Finally, the numerical predictions from our model are compared with the published experimental results and found to be in close agreement.
\end{abstract}

Keywords-2DEG-two dimensional electron gas, DC-HEMT-dual channel high electron mobility transistor, MODFET- modulation doped field effect transistor, UID-unintentionally doped.

\section{INTRODUCTION}

In present senerio $\mathrm{GaN}$ based hetrostructure devices are emerging as an important option for microwave frequencies and high power applications in advanced electronic system operating at higher voltages. A great amount of research work has already been done on $\mathrm{AlGaAs} / \mathrm{GaAs}$ devices for optimization and performace enhancement, still AlGaN/GaN High Electron Mobility Transistor (HEMT) or Modulation Doped Field Effect Transistor (MODFET) are attracting considerable attention of device scintists and engineers world wide for further innovations and optimizations. Better performance of $\mathrm{AlGaN} / \mathrm{GaN}$ material system based hetrostructures relies on its high saturation velocity, thermal stability, high breakdown electric field and unique property of polarization and piezoelectric induced high sheet charge density of 2-DEG at hetrointerface [1]. The $\mathrm{GaN}$ material system based optimized hetrostructures can withstand much higher output power density at considerably higher operating voltages as compared with conventional AlGaAs/GaAs-HEMT [2]-[3]. Long et al [4]-[5] has proposed a FET structure, the dual material gate FET, in which two different metals of different workfunction were used to engineer a single gate by making them contact laterally. An epilayer structure of $\mathrm{AlGaN} / \mathrm{GaN}$ double channel High Electron Mobility Transistor was fabricated and characterised by Chu et.al. [6] resulting in to device with high current gain and minimized current collapse. Recently, detailed analytical models have been presented for analysing large signal parameters, transconductance, electric potential and electric field distribution along the channels of Double Channel Dual Material Gate $\mathrm{Al}_{\mathrm{m}} \mathrm{Ga}_{1-\mathrm{m}} \mathrm{N} / \mathrm{GaN}$ High Electron Mobility Transistor [19][20]. Also a current voltage and transconductance model for dual material gate $\mathrm{Al}_{\mathrm{m}} \mathrm{Ga}_{1}$ ${ }_{\mathrm{m}} \mathrm{N} / \mathrm{GaN}$ modulation doped field effect transistor presented for analysis of dc characteristics of the device strcture [21].

In our present paper, we proposed 2-D model for I-V characteristics and transconductance of dual channel AlGaN/GaN MODFET. In past considerable research have been done for enhancing the device current drive capability and also double channel AlGaN/GaN MODFET was fabricated. For incorporation of double channel effects, there was requirement of additional $\mathrm{AlGaN}$ barrier layer between the two channels to supply carriers for bottom channel. The additional layer of AlGaN reduced accesses to the lower channel thus the double channel behaviors was not pronounced effectively. First time Rongming Chu et al [6] have shown that this kind of problem could be resolved by efficient design of double channel structures. Construction of an $\mathrm{AlGaN} / \mathrm{GaN} / \mathrm{AlGaN} / \mathrm{GaN}$ multi layer structure using a lower AlGaN barrier layer with small layer thickness and graded Al composition was proved to be an effective approach. Their design resulted in to the double-channel MODFET with the second channel of considerably high sheet 
carrier density and acceptable access resistance. Since fabrication of dual channel AlGaN/GaN HEMT, so far no analytical model is developed to explain the effect of double channel MODFET. Our paper is an effort for derivation of I$\mathrm{V}$ characteristics and transconductance 2-D analytical model for dual channel MODFETs.

\section{MODEL FORMULATION}

Fig. 1 shows the schematic crosssectional view of structure of dual channel $\mathrm{Al}_{0.3} \mathrm{Ga}_{0.7} \mathrm{~N} / \mathrm{GaN}$ MODFET, where $\mathrm{L}$ is length of the gate metal of length $1 \mu \mathrm{m}$. The gate electrode is made of Au forming schottky junction between metal and AlGaN cap layer of $3 \mathrm{~nm}$ thickness followed by Si doped barrier layer of $18 \mathrm{~nm}$ thickness. After barrier layer a spacer layer of $3 \mathrm{~nm}$ thickness is used in between to reduce impurity scattering then a $14 \mathrm{~nm}$ thick $\mathrm{GaN}$ channel layer is formed for creating 2DEG channel in side it. After upper channel layer a graded profile $(\mathrm{m}=3 \%$ to $6 \%)$ barrier layer of thickness $21 \mathrm{~nm}$ follows.

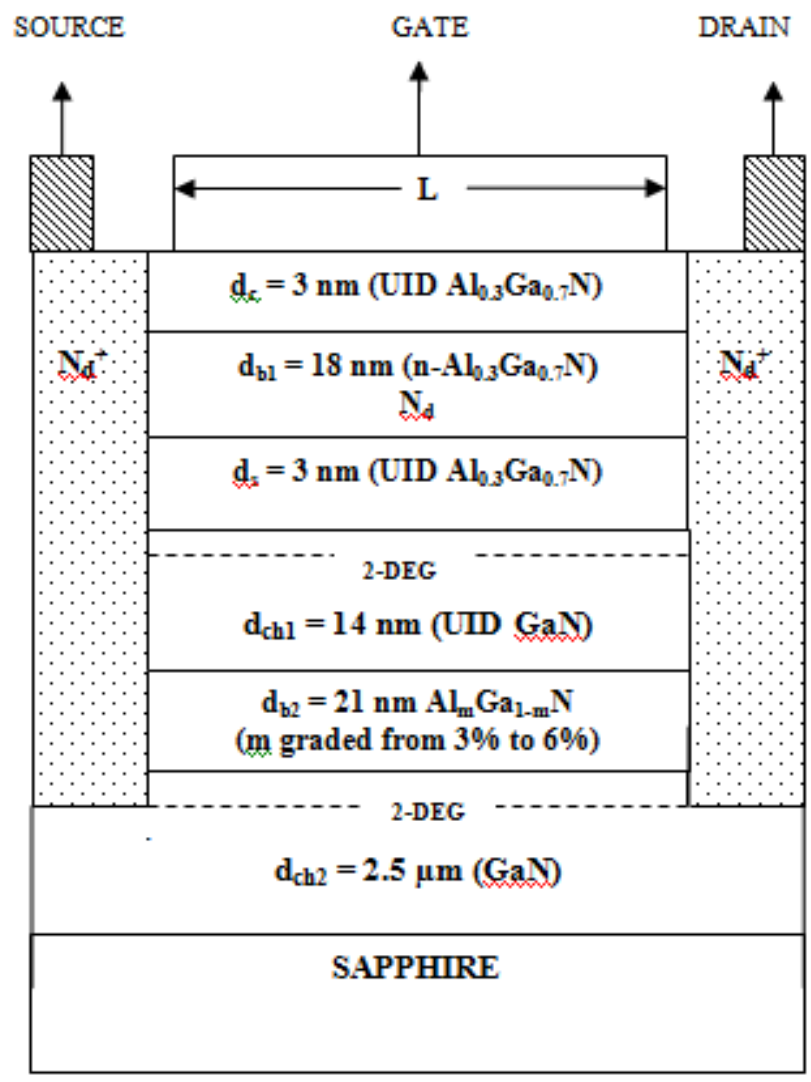

Fig.1. Two dimensional structure of Dual Channel AlGaN/GaN MODFET epilayer on sapphire substrate based on [6], total gate length $(\mathrm{L})=1 \mu \mathrm{m}$, work function of gate region $\mathrm{M}\left(\phi_{\mathrm{M}}\right)=5.3 \mathrm{~V}$, Gate width $(\mathrm{W})=100 \mu \mathrm{m}$, $\mathrm{N}_{\mathrm{d}}=2 \times 10^{24} \mathrm{~m}^{-3}, \mathrm{~N}_{\mathrm{d}}^{+}=1 \times 10^{26} \mathrm{~m}^{-3}$

Due to optimum thickness of barrier layer it is assumed that Si doped $\mathrm{Al}_{0.3} \mathrm{Ga}_{0.7} \mathrm{~N}$ layer is fully depleted under normal operating conditions. It is also assumed that all electons as charge carriers are confined in top and bottom channels and forming 2-DEG for each channel.
Finally $2.5 \mu \mathrm{m}$ GaN layer is grown on sapphire substrate to accommodate bottom channel. The metal gate can control charge in the top and bottom conducting channels formed at hetrointerfaces of $\mathrm{Al}_{0.3} \mathrm{Ga}_{0.7} \mathrm{~N} / \mathrm{GaN} / \mathrm{Al}_{\mathrm{m}} \mathrm{Ga}_{1-\mathrm{m}} \mathrm{N} / \mathrm{GaN}$ epilayers in the device. In double channel device structure the source and drain contacts regions are uniformally doped with higher doping profile of $\mathrm{N}_{\mathrm{d}}^{+}=10^{26} \mathrm{~m}^{-3}$ to ensure that these terminals behave as ohmic contacts.

For our depletion enhancement mode (normally on) HEMT device the Si-doped barrier layers are fully depleted without applying any gate bias. In this condition 2 DEG based conducting channel is also formed for current conduction in the device. Under influence of an applied external bias at the gate terminal, the $\mathrm{n}-\mathrm{Al}_{\mathrm{m}} \mathrm{Ga}_{1-\mathrm{m}} \mathrm{N}$ barrier layer is depleted of charge carriers partially at the hetrointerfaces due to electron diffision into the channels and partially at the surface due to presence of Schottky barrier [7][8][9]. For complete charge modulation by the gate terminal, each channel regions must completely overlap to deplete the n-AlGaN layers so that no parasitic conduction path exists in device and only 2-DEG high mobility electrons take part in current conduction process in top and bottom channels.

Considering spontaneous and pizoelectric polarization at the hetrointerface the sheet carrier concentration of 2-DEG in upper channels is obtained [15] as

$$
n_{s 1}(m, x)=\frac{\epsilon(m)}{q d_{1}}\left(V_{g s}-V_{t h 1}(m)-V_{c 1}(x)\right) \text { for } 0 \leq x \leq
$$

With $\boldsymbol{\epsilon}(\boldsymbol{m})$ as $\mathrm{Al}_{\mathrm{m}} \mathrm{Ga}_{1-\mathrm{m}} \mathrm{N}$ dielectric constant, and $\mathrm{m}=0.3$ is $\mathrm{Al}$ mole fraction in it, $\mathrm{V}_{\mathrm{cl}}(\mathrm{x})$ as upper channel potential at any point $\mathrm{x}$ along the upper channel, $\mathrm{V}_{\mathrm{gs}}$ as gate to source bias, $\mathrm{d}_{1}=\mathrm{d}_{\mathrm{b} 1}+\mathrm{d}_{\mathrm{s}}+\mathrm{d}_{\mathrm{c}}+\Delta \mathrm{d}_{1}$ as separation between gate and upper channel and $\Delta \mathrm{d}_{1}$ depth of $2 \mathrm{DEG}$ in upper $\mathrm{GaN}$ layer from its top edge.

Similarily, the sheet carrier concentration of 2-DEG in lower channels is obtained as

$$
\begin{gathered}
n_{s 2}(m, x)=\frac{\epsilon(m)}{q d_{2}}\left(V_{g s}-V_{t h 2}(m)-V_{c 2}(x)\right) \text { for } 0 \leq \\
x \leq L(2)
\end{gathered}
$$

Where $\mathrm{V}_{\mathrm{c} 2}(\mathrm{x})$ is channels potential at any point $\mathrm{x}$ along the lower channel and $\mathrm{d}_{2}=\mathrm{d}_{1}+\mathrm{d}_{\mathrm{ch} 1}+\mathrm{d}_{\mathrm{b} 2}+\Delta \mathrm{d}_{2}$ is separation between gate and lower channel where $\Delta \mathrm{d}_{2}$ depth of 2DEG in lower $\mathrm{GaN}$ layer from its top edge.

The device threshold voltages for upper and lower channels are obtained [10] as

$$
\begin{aligned}
& V_{t h 1}(m)=\epsilon(m)-\Delta \phi(m)-\frac{q\left(N_{d}-N_{t}\right) d_{b 1}^{2}}{2 \epsilon(m)}-\frac{\sigma_{p z}(m) d_{1}}{\epsilon(m)} \\
& V_{t h 2}(m)=\epsilon(m)-\Delta \phi(m)-\frac{q\left(N_{d}-N_{t}\right) d_{b 2}^{2}}{2 \epsilon(m)}-\frac{\sigma_{p z}(m) d_{2}}{\epsilon(m)}
\end{aligned}
$$


where $\boldsymbol{\epsilon}(\boldsymbol{m})$ as the Schottky barrier height, $\boldsymbol{\Delta} \boldsymbol{\phi}(\boldsymbol{m})$ as the conduction band discontinuity at the $\mathrm{Al}_{\mathrm{m}} \mathrm{Ga}_{1-\mathrm{m}} \mathrm{N} / \mathrm{GaN}$ hetrointerface. $\mathrm{N}_{\mathrm{d}}$ is $\mathrm{Si}$ doping concentration of $\mathrm{n}$-AlGaN layer and $\boldsymbol{N}_{\boldsymbol{t}}$ is trap concentration of surface states, $\boldsymbol{\sigma}_{\boldsymbol{p} \boldsymbol{z}}(\boldsymbol{m})$ net polarization induced sheet carrier density at the hetrointerfaces and obtained as

$$
\sigma_{p z}(m)=\left[P_{S P}(m)-P_{S P}(0)+P_{p z}\right]
$$

Where $\mathrm{P}_{\mathrm{SP}}(\mathrm{m})$ and $\mathrm{P}_{\mathrm{SP}}(0)$ is spontaneous polarization of $\mathrm{Al}_{\mathrm{m}} \mathrm{Ga}_{1-\mathrm{m}} \mathrm{N}$ and $\mathrm{GaN}$ material system[1][11][12].

In our model piezoelectric polarization depen on the amount of strain developed at hetrointerfaces in order to accommodate the difference in lattice constants of $\mathrm{GaN}$ and AlGaN. For fully satrained device piezoelectric polarization dependent charge density is given as

$P_{p z}=2\left(\frac{a(0)-a(m}{a(m)}\right) \quad\left(e_{31}(m)-e_{33}(m) \frac{c_{13}(m)}{C_{33}(m)}\right) \quad$ for $0 \leq \mathrm{m} \leq 1$

$$
\begin{aligned}
\left|\sigma_{p z}(m)\right|=\mid 2 & \left(\frac{a(0)-a(m)}{a(m)}\right)\left(e_{31}(m)\right. \\
& \left.-e_{33}(m) \frac{C_{13}(m)}{C_{33}(m)}\right)+P_{S P}(m) \\
& -P_{S P}(0) \mid
\end{aligned}
$$

(6)

for $\quad 0 \leq \mathrm{m} \leq 1$

Whre $\mathrm{a}(\mathrm{m})$ is lattice constant $\mathrm{e}_{31}(\mathrm{~m})$ and $\mathrm{e}_{32}(\mathrm{~m})$ are piezoelecric constants and $\mathrm{C}_{13}(\mathrm{~m})$ and $\mathrm{C}_{33}(\mathrm{~m})$ are elastic constants [12][13].

When we increase $\mathrm{Al}$ mole fraction in barrier layer it results in increase in lattice mismatch that further causes in strain relaxation. In a partially relaxed device with $\mathrm{Al}_{\mathrm{m}} \mathrm{Ga}_{1-\mathrm{m}} \mathrm{N}$ layer thickness between $18 \mathrm{~nm}$ to $40 \mathrm{~nm}$, the piezoelectric polarization induced charge density obtained as [14].

$$
\begin{aligned}
& P_{P z}= \\
& \left\{\begin{array}{rr}
2\left(\frac{a(0)-a(m}{a(m)}\right) & \left(e_{31}(m)-e_{33}(m) \frac{C_{13}(m)}{C_{33}(m)}\right) \\
2\left(\frac{2.33-3.5 m}{\frac{a(0)-a(m}{a(m)}}\right) & \left(e_{31}(m)-e_{33}(m) \frac{c_{13}(m)}{C_{33}(m)}\right) \\
0 & \text { for } 0.38 \leq m \leq 0.67
\end{array}\right. \\
&
\end{aligned}
$$

The above expression gives approximate range for which a GaN/AlGaN material system based hetreointerface remains completely strained and partially relaxed.

Table.1 Material parameters of $\mathrm{Al}_{\mathrm{m}} \mathrm{Ga}_{1-\mathrm{m}} \mathrm{N} / \mathrm{GaN}$ for $\mathrm{m}=0.30$

\begin{tabular}{lll}
\hline $\mathrm{R}_{\mathrm{d}}$ & Parasitic Drain Resistance & $3 \mathrm{Ohm}$ \\
$\mathrm{R}_{\mathrm{s}}$ & Parasitic Source Resistance & $3 \mathrm{Ohm}$ \\
$\mathrm{a}(0)$ & lattice constant & $3.1890 \dot{\mathrm{A}}$ \\
$\mathrm{a}(\mathrm{m})$ & lattice constant & $3.1659 \dot{\mathrm{A}}$
\end{tabular}

$\mathrm{e}_{31}(\mathrm{~m})$ piezoelectric constant $(\mathrm{x}, \mathrm{y})$

$-0.523 \mathrm{C} / \mathrm{m}^{2}$

$\mathrm{e}_{33}(\mathrm{~m})$ piezoelectric constant $(\mathrm{z})$

$0.949 \mathrm{C} / \mathrm{m}^{2}$

$\mathrm{C}_{13}(\mathrm{~m})$ elastic constant $(\mathrm{x}, \mathrm{y})$

$104.5 \mathrm{G} \mathrm{Pa}$

$\mathrm{C}_{33}(\mathrm{~m})$ elastic constant (z)

$395.4 \mathrm{G} \mathrm{Pa}$

$\left.\mathrm{P}_{\mathrm{SP}}(0)\right)$ spontaneous polarization $\mathrm{GaN}$

$-0.029 \mathrm{C} / \mathrm{m}^{2}$

$\left.\mathrm{P}_{\mathrm{SP}}(\mathrm{m})\right)$ spontaneous polarization $\mathrm{Al}_{\mathrm{m}} \mathrm{Ga}_{1-\mathrm{m}} \mathrm{N}-0.038 \mathrm{C} / \mathrm{m}^{2}$

$\mu_{0} \quad$ low field mobility $\quad 370 \times 10^{-4} \mathrm{~m}^{2} / \mathrm{V} . \mathrm{s}$

$E_{c} \quad$ Critical Electrical Field $\quad 190 \times 10^{5} \mathrm{~V} / \mathrm{m}$

$v_{s} \quad$ Electron saturation drift velocity $2.1 \times 10^{5} \mathrm{~m} / \mathrm{s}$

$\Delta \mathrm{d} \quad 2$ DEG depth in GaN $10 \mathrm{~nm}$

\subsection{Current Voltage Characteristics}

Linear Region Model: Double channel device current can be obtained using current density equation for upper and lower channel as

$I_{d 1}(m, x)=Z q \mu(x)\left(n_{s 1}(m, x) \frac{d V_{c 1}(x)}{d x}+\frac{k T}{q} \frac{d n_{s 1}(m, x)}{d x}\right)$

$I_{d 2}(m, x)=Z q \mu(x)\left(n_{s 2}(m, x) \frac{d^{2} 2(x)}{d x}+\frac{k T}{q} \frac{d_{s 2}(m, x)}{d x}\right)$

where $\mathrm{Z}$ is gate width, $\mathrm{V}_{\mathrm{c} 1}(\mathrm{x})$ and $\mathrm{V}_{\mathrm{c} 2}(\mathrm{x})$ are potential in upper and lower channels along $\mathrm{x}$ axis respectively, $\mathrm{q}$ is electron charge, $\mathrm{k}$ is Boltzmann constant, $\mathrm{T}$ is ambient temperature. The field dependent electron mobility in each channel is obtained [16][17] as

$\mu_{1}(x)=\frac{\mu_{0}}{1+\left(\frac{\mu_{0} E_{c}-v_{S}}{E_{c} v_{S}}\right) \frac{\mathrm{dV} V_{c}(\mathrm{x})}{\mathrm{dx}}}$

$\mu_{2}(x)=\frac{\mu_{0}}{1+\left(\frac{\mu_{0} E_{c}-v_{s}}{E_{c} v_{S}}\right) \frac{\mathrm{dV} c 2(\mathrm{x})}{\mathrm{dx}}}$

Where, $\mu_{0}$ is low field mobility, $E_{c}$ is critical electric field, and $v_{s}$ is saturation drift velocity of the electrons.

Using (1) and (10) in (8) we obtain

$$
\begin{gathered}
\mathrm{I}_{\mathrm{d} 1}(\mathrm{~m}, \mathrm{x})\left(1+\left(\frac{\mu_{0} E_{c}-v_{s}}{E_{c} v_{s}}\right) \frac{\mathrm{dV}_{\mathrm{c} 1}(\mathrm{x})}{\mathrm{dx}}\right) \\
\mathrm{Z} \mu_{0} \frac{\epsilon(\mathrm{m})}{\mathrm{d}_{1}}\left(\left(\mathrm{~V}_{\mathrm{f} 1}(\mathrm{~m})-\mathrm{V}_{\mathrm{c} 1}(\mathrm{x})\right) \frac{\mathrm{dV}_{\mathrm{c} 1}(\mathrm{x})}{\mathrm{dx}}\right)
\end{gathered}
$$

Similarly, using (2) and (11) in (9) we obtain

$$
\begin{gathered}
\mathrm{I}_{\mathrm{d} 2}(\mathrm{~m}, \mathrm{x})\left(1+\left(\frac{\mu_{0} E_{c}-v_{s}}{E_{c} v_{s}}\right) \frac{\mathrm{dV}_{\mathrm{c} 2}(\mathrm{x})}{\mathrm{dx}}\right)= \\
\mathrm{Z} \mu_{0} \frac{\epsilon(\mathrm{m})}{\mathrm{d}_{2}}\left(\left(\mathrm{~V}_{\mathrm{f} 2}(\mathrm{~m})-\mathrm{V}_{\mathrm{c} 2}(\mathrm{x})\right) \frac{\mathrm{dV}_{\mathrm{c} 2}(\mathrm{x})}{\mathrm{dx}}\right)
\end{gathered}
$$

Where

$$
\begin{aligned}
& V_{f 1}=V_{g s}-V_{t h 1}(m)-\frac{k T}{q} \\
& V_{f 2}=V_{g s}-V_{t h 2}(m)-\frac{k T}{q}
\end{aligned}
$$

Integrating (12) and (13) with boundary conditions for each channel at $\mathrm{x}=0$ 


$$
\begin{aligned}
& \left.V_{c 1}(x)\right|_{x=0}=\mathrm{I}_{\mathrm{d} 1}(\mathrm{~m}) R_{s} \\
& \left.V_{c 2}(x)\right|_{x=0}=\mathrm{I}_{\mathrm{d} 2}(\mathrm{~m}) R_{s}
\end{aligned}
$$

And

$$
\begin{aligned}
& \left.V_{c 1}(x)\right|_{x=L}=V_{d s}-\mathrm{I}_{\mathrm{d} 1}(\mathrm{~m})\left(R_{s}+R_{d}\right) \\
& \left.V_{c 2}(x)\right|_{x=L}=V_{d s}-\mathrm{I}_{\mathrm{d} 2}(\mathrm{~m})\left(R_{s}+R_{d}\right)
\end{aligned}
$$

Where, $L$ is gate length, $V_{d s}$ is applied drain bias, $R_{d}$ and $R_{s}$ are parasitic source and drain resistances respectively. The current equation for upper channel is obtained as

$$
I_{d 1}(m)=\frac{-\alpha_{2}(\mathrm{~m}) \pm \sqrt{\alpha_{2}(\mathrm{~m})^{2}-4 \alpha_{1}(\mathrm{~m}) \alpha_{3}(\mathrm{~m})}}{2 \alpha_{1}(\mathrm{~m})}
$$

Where,

$$
\begin{gathered}
\alpha_{1}(\mathrm{~m})=\left(\frac{\mu_{0} E_{c}-v_{s}}{\mathrm{E}_{\mathrm{c}} \mathrm{v}_{\mathrm{s}}}\right)\left(2 \mathrm{R}_{\mathrm{s}}+\mathrm{R}_{\mathrm{d}}\right) \\
-\left(\frac{\mathrm{Z} \mu_{0} \epsilon(\mathrm{m}}{2 \mathrm{~d}_{1}}\right)\left(\mathrm{R}_{\mathrm{d}}^{2}+2 \mathrm{R}_{\mathrm{s}} \mathrm{R}_{\mathrm{d}}\right)
\end{gathered}
$$

$$
\begin{aligned}
& \alpha_{2}(\mathrm{~m})=\left(\frac{\mathrm{Z} \mu_{0} \epsilon(\mathrm{m}}{\mathrm{d}_{1}}\right)\left(\mathrm{V}_{\mathrm{ds}}\left(\mathrm{R}_{\mathrm{s}}+\mathrm{R}_{\mathrm{d}}\right)+\mathrm{V}_{\mathrm{f} 1}(\mathrm{~m})\left(2 \mathrm{R}_{\mathrm{s}}+\mathrm{R}_{\mathrm{d}}\right)\right)- \\
& \mathrm{L}-V_{d s}\left(\frac{\mu_{0} E_{c}-v_{s}}{\mathrm{E}_{\mathrm{c}} \mathrm{v}_{\mathrm{s}}}\right) \\
& \alpha_{3}(\mathrm{~m})=\left(\frac{\mathrm{Z} \mu_{0} \epsilon(\mathrm{m}}{\mathrm{d}_{1}}\right)\left(\mathrm{V}_{\mathrm{f} 1}(\mathrm{~m}) \mathrm{V}_{\mathrm{ds}}-\frac{\mathrm{V}_{\mathrm{ds}}^{2}}{2}\right)
\end{aligned}
$$

Similarly, current equation for lower channel is given by

$$
I_{d 2}(m)=\frac{-\beta_{2}(\mathrm{~m}) \pm \sqrt{\beta_{2}(\mathrm{~m})^{2}-4 \beta_{1}(\mathrm{~m}) \beta_{3}(\mathrm{~m})}}{2 \beta_{1}(\mathrm{~m})}
$$

Where,

$$
\begin{gathered}
\beta_{1}(\mathrm{~m})=\left(\frac{\mu_{0} E_{c}-v_{s}}{\mathrm{E}_{\mathrm{c}} \mathrm{v}_{\mathrm{s}}}\right)\left(2 \mathrm{R}_{\mathrm{s}}+\mathrm{R}_{\mathrm{d}}\right) \\
-\left(\frac{\mathrm{Z} \mu_{0} \epsilon(\mathrm{m}}{2 \mathrm{~d}_{2}}\right)\left(\mathrm{R}_{\mathrm{d}}^{2}+2 \mathrm{R}_{\mathrm{s}} \mathrm{R}_{\mathrm{d}}\right) \\
(25) \\
\beta_{2}(\mathrm{~m})=\left(\frac{\mathrm{Z} \mu_{0} \epsilon(\mathrm{m}}{\mathrm{d}_{2}}\right)\left(\mathrm{V}_{\mathrm{ds}}\left(\mathrm{R}_{\mathrm{s}}+\mathrm{R}_{\mathrm{d}}\right)+\mathrm{V}_{\mathrm{f} 2}(\mathrm{~m})\left(2 \mathrm{R}_{\mathrm{s}}+\mathrm{R}_{\mathrm{d}}\right)\right)- \\
\mathrm{L}-V_{d s}\left(\frac{\mu_{0} E_{c}-v_{s}}{\mathrm{E}_{\mathrm{c}} \mathrm{v}_{\mathrm{s}}}\right) \\
\beta_{3}(\mathrm{~m})=\left(\frac{\mathrm{Z} \mu_{0} \epsilon(\mathrm{m}}{\mathrm{d}_{2}}\right)\left(\mathrm{V}_{\mathrm{f} 2}(\mathrm{~m}) \mathrm{V}_{\mathrm{ds}}-\frac{\mathrm{v}_{\mathrm{ds}}^{2}}{2}\right)
\end{gathered}
$$

Thus, device current for linear region is obtained by summation of both channels' linear region currents, as

$$
I_{d}(m)=I_{d 1}(m)+I_{d 2}(m)
$$

Saturation Region Model: In saturation region, at onset of saturation the potential in both the channels attains drain saturation voltage, $\mathrm{V}_{\mathrm{dsat} 1}(\mathrm{~m})$ and electric field attains critical value $\mathrm{E}_{\mathrm{c}}$. The current in saturation region in upper channel, $\mathrm{I}_{\mathrm{dsat} 1}$ can be obtained from equation (20) by substituting $\mathrm{V}_{\mathrm{ds}}$ by $\mathrm{V}_{\mathrm{dsat} 1}(\mathrm{~m})$ obtained as

$\mathrm{V}_{\mathrm{dsat} 1}(\mathrm{~m})=\frac{-\omega_{2}(\mathrm{~m}) \pm \sqrt{\omega_{2}(m)^{2}-4 \omega_{1}(\mathrm{~m}) \omega_{3}(\mathrm{~m})}}{2 \omega_{1}(\mathrm{~m})}$

Where,

$$
\begin{gathered}
\omega_{1}(\mathrm{~m})=\theta(m)+\left(\frac{\mathrm{Z} \mu_{0} \epsilon(\mathrm{m}}{\mathrm{d}_{1}}\right)\left(\left(\frac{\mu_{0} E_{c}-v_{s}}{\mathrm{E}_{\mathrm{c}} \mathrm{v}_{\mathrm{s}}}\right)-\frac{1}{2}\right)- \\
\left(\frac{\mathrm{Z} \mu_{0} \epsilon(\mathrm{m})}{\mathrm{d}_{1}}\right)^{2} E_{c}\left(\mathrm{R}_{\mathrm{s}}+\mathrm{R}_{\mathrm{d}}\right) \\
\left(\frac{\mathrm{Z} \mu_{0} \epsilon(\mathrm{m}}{\mathrm{d}_{1}}\right)\left(\frac{\mu_{0} E_{c} \mathrm{~V}_{\mathrm{f} 1}(\mathrm{~m})}{\mathrm{v}_{\mathrm{s}}}+E_{c} L\right)- \\
2 \mathrm{~V}_{\mathrm{f} 1}(\mathrm{~m}) \theta(m)+\left(\frac{\mathrm{Z} \mu_{0} \epsilon(\mathrm{m})}{\mathrm{d}_{1}}\right)^{3} E_{c} \mathrm{~V}_{\mathrm{f} 1}(\mathrm{~m})\left(3 \mathrm{R}_{\mathrm{s}}+2 \mathrm{R}_{\mathrm{d}}\right) \\
\omega_{3}(\mathrm{~m})=\theta(m) \mathrm{V}_{\mathrm{f} 1}^{2}(\mathrm{~m})-\left(\frac{\mathrm{Z} \mu_{0} \epsilon(\mathrm{m}}{\mathrm{d}_{1}}\right) \mathrm{V}_{\mathrm{f} 1}(\mathrm{~m}) E_{c} L- \\
\left(\frac{\mathrm{Z} \mu_{0} \epsilon(\mathrm{m})}{\mathrm{d}_{1}}\right)^{3} \mathrm{~V}_{\mathrm{f} 1}^{2}(\mathrm{~m}) E_{c}\left(2 \mathrm{R}_{\mathrm{s}}+\mathrm{R}_{\mathrm{d}}\right)
\end{gathered}
$$

Where,

$$
\begin{aligned}
& \theta(m)=\left(\frac{\mathrm{Z} \mu_{0} \epsilon(\mathrm{m}) E_{c}}{\mathrm{~d}_{1}}\right)^{2}\left(\frac{\mu_{0} E_{c}-v_{s}}{\mathrm{E}_{\mathrm{c}} \mathrm{v}_{\mathrm{s}}}\right)\left(2 \mathrm{R}_{\mathrm{s}}+\mathrm{R}_{\mathrm{d}}\right)- \\
& \left(\frac{\mathrm{Z} \mu_{0} \epsilon(\mathrm{m})}{\mathrm{d}_{1}}\right)^{3} \frac{E_{c}^{2}}{2}\left(\mathrm{R}_{\mathrm{d}}^{2}+2 \mathrm{R}_{\mathrm{s}} \mathrm{R}_{\mathrm{d}}\right)
\end{aligned}
$$

Similarly, the current in saturation region in lower channel, $\mathrm{I}_{\mathrm{dsat} 2}$ can be obtained from (24) by substituting $\mathrm{V}_{\mathrm{ds}}$ by $\mathrm{V}_{\text {dsat } 2}(\mathrm{~m})$ obtained as

$$
\mathrm{V}_{\mathrm{dsat} 2}(\mathrm{~m})=\frac{-\delta_{2}(m) \pm \sqrt{\delta_{2}(m)^{2}-4 \delta_{1}(\mathrm{~m}) \delta_{2}(m)}}{2 \delta_{1}(\mathrm{~m})}
$$

Where,

$$
\begin{gathered}
\delta_{1}(\mathrm{~m})=\lambda(m)+\left(\frac{\mathrm{Z} \mu_{0} \epsilon(\mathrm{m})}{\mathrm{d}_{2}}\right)\left(\left(\frac{\mu_{0} E_{c}-v_{s}}{\mathrm{E}_{\mathrm{c}} \mathrm{v}_{\mathrm{s}}}\right)-\frac{1}{2}\right)- \\
\left(\frac{\mathrm{Z} \mu_{0} \epsilon(\mathrm{m})}{\mathrm{d}_{2}}\right)^{2} E_{c}\left(\mathrm{R}_{\mathrm{s}}+\mathrm{R}_{\mathrm{d}}\right) \\
\delta_{2}(m)= \\
\left.2 \frac{\mathrm{Z} \mu_{0} \epsilon(\mathrm{m})}{\mathrm{d}_{2}}\right)\left(\frac{\mu_{0} E_{c} \mathrm{~V}_{\mathrm{f} 2}(\mathrm{~m})}{\mathrm{v}_{\mathrm{s}}}+E_{c} L\right)- \\
\delta_{\mathrm{f} 2}(\mathrm{~m}) \lambda(m)+\left(\frac{\mathrm{Z} \mu_{0} \epsilon(\mathrm{m})}{\mathrm{d}_{2}}\right)^{3} E_{c} \mathrm{~V}_{\mathrm{f} 2}(\mathrm{~m})\left(3 \mathrm{R}_{\mathrm{s}}+2 \mathrm{R}_{\mathrm{d}}\right) \\
\left(\frac{\mathrm{Z} \mu_{0} \epsilon(\mathrm{m})}{\mathrm{d}_{2}}\right)^{3} \mathrm{~V}_{\mathrm{f} 2}^{2}(\mathrm{~m}) E_{c}\left(2 \mathrm{R}_{\mathrm{s}}+\mathrm{R}_{\mathrm{d}}\right)
\end{gathered}
$$

Where,

$$
\begin{aligned}
& \lambda(m)=\left(\frac{\mathrm{Z} \mu_{0} \epsilon(\mathrm{m}) E_{c}}{\mathrm{~d}_{2}}\right)^{2}\left(\frac{\mu_{0} E_{c}-v_{s}}{\mathrm{E}_{\mathrm{c}} \mathrm{v}_{\mathrm{s}}}\right)\left(2 \mathrm{R}_{\mathrm{s}}+\mathrm{R}_{\mathrm{d}}\right)- \\
& \left(\frac{\mathrm{Z} \mu_{0} \epsilon(\mathrm{m})}{\mathrm{d}_{2}}\right)^{3} \frac{E_{c}{ }^{2}}{2}\left(\mathrm{R}_{\mathrm{d}}^{2}+2 \mathrm{R}_{\mathrm{s}} \mathrm{R}_{\mathrm{d}}\right)
\end{aligned}
$$


Thus, device current in saturation region is obtained as

$$
\mathrm{I}_{\mathrm{dsat}}(\mathrm{m})=\mathrm{I}_{\mathrm{dsat} 1}(\mathrm{~m})+\mathrm{I}_{\mathrm{dsat} 1}(\mathrm{~m})
$$

\subsection{Transfer Characteristics}

Transfer characteristics is relationship with variation of drain current $I_{d}(m)$ with respect to variation in gate to source voltage, $V_{\mathrm{gs}}$ at constant drain to source voltage. It is obtained as

$$
\left.\frac{I_{d}(m)}{\mathrm{V}_{\mathrm{gs}}}\right|_{V_{d s=\text { constant }}}
$$

Device Trans-conductance: This parameter is important for prediction of microwave performances and analysis of double channel $\mathrm{Al}_{\mathrm{m}} \mathrm{Ga}_{1-\mathrm{m}} \mathrm{N} / \mathrm{GaN}$ HEMT high frequency behavior. This parameter is evaluated for upper channel as

$$
g_{m 1}(m)=\left.\frac{\partial I_{d 1}(m)}{\partial V_{d s}}\right|_{V d s=\text { constant }}
$$

Using (20) in (40) we obtain upper channel transconductance in linear region $g_{m 1}(m)$ as

$$
\begin{gathered}
g_{m 1}(m)= \\
\left(\frac{\mathrm{Z} \mu_{0} \epsilon(\mathrm{m})}{\mathrm{d}_{1}}\right)\left(\frac{2 \mathrm{R}_{\mathrm{s}}+\mathrm{R}_{\mathrm{d}}}{2 \alpha_{1}(\mathrm{~m})}\left(1-\alpha_{2}(\mathrm{~m})\right)-\frac{\mathrm{V}_{\mathrm{ds}}}{\sqrt{\alpha_{2}(\mathrm{~m})^{2}-4 \alpha_{1}(\mathrm{~m}) \alpha_{3}(\mathrm{~m})}}\right)(41)
\end{gathered}
$$

Trans-conductance for lower channel is evaluated as

$$
g_{m 2}(m)=\left.\frac{\partial I_{d 2}(m)}{\partial V_{d s}}\right|_{V d s}
$$

Similarly using (24) in (42) we obtain upper channel transconductance in linear region $g_{m 2}(m)$ as

$$
\begin{gathered}
g_{m 2}(m)= \\
\left(\frac{\mathrm{Z} \mu_{0} \epsilon(\mathrm{m})}{\mathrm{d}_{2}}\right)\left(\frac{2 \mathrm{R}_{\mathrm{s}}+\mathrm{R}_{\mathrm{d}}}{2 \beta_{1}(\mathrm{~m})}\left(1-\beta_{2}(\mathrm{~m})\right)-\frac{\mathrm{V}_{\mathrm{ds}}}{\sqrt{\beta_{2}(\mathrm{~m})^{2}-4 \beta_{1}(\mathrm{~m}) \beta_{3}(\mathrm{~m})}}\right)
\end{gathered}
$$

The device trans-conductance $g_{m}(m)$ is obtained as

$$
g_{m}(m)=g_{m 1}(m)+g_{m 2}(m)
$$

\section{RESULTS AND DISCUSSIONS}

In order to verify our derived $2 \mathrm{D}$ model for current voltage characteristics and transconductance of dual channel AlGaN/GaN MODFET, the results obtained from numerical calculations have been compared with published experimental data of fabricated device [6]. Following Fig. 2 depicts the cumulative current of both upper channel and lower channel as obtained from the analytical model and from experimental data. In this figure, the dash lines represent the experimental results while the solid lines display model data for various gate to source bias conditions. Ploted results gives output characteristics of device in both linear and saturation regions of device operation at various gate to source biases starting at $1 \mathrm{~V}$ with a step of $-1 \mathrm{~V}$. The drain current of the device is increasing from approximately $470 \mathrm{~mA} / \mathrm{mm}$ to $850 \mathrm{~mA} / \mathrm{mm}$ with corresponding increments in gate to source bias. On comparison we can say that plotted results are in good agreement wih reported experimental data. In our proposed model, the cumulative drain current is found to vary with the distance of each of the channel from the gate surface, depicting the current collapse in each channel is dependent on channel distance from the device surface. Current collapse also depends on trapping and detarpping of surface states in device structure which is modelled in our device threshold expressions for each channel. Device threshold voltage can be calculated from threshold expressions as stated above. It was revealed from analysis of results that more current collapse occuers in the upper channel closer to the device surface, whereas the lower channel suffers minimal current collapse being at far distance. Expressions also show that trapping/de-trapping of surface states is also responsible for current collapse in GaN based dual channel MODFETs.

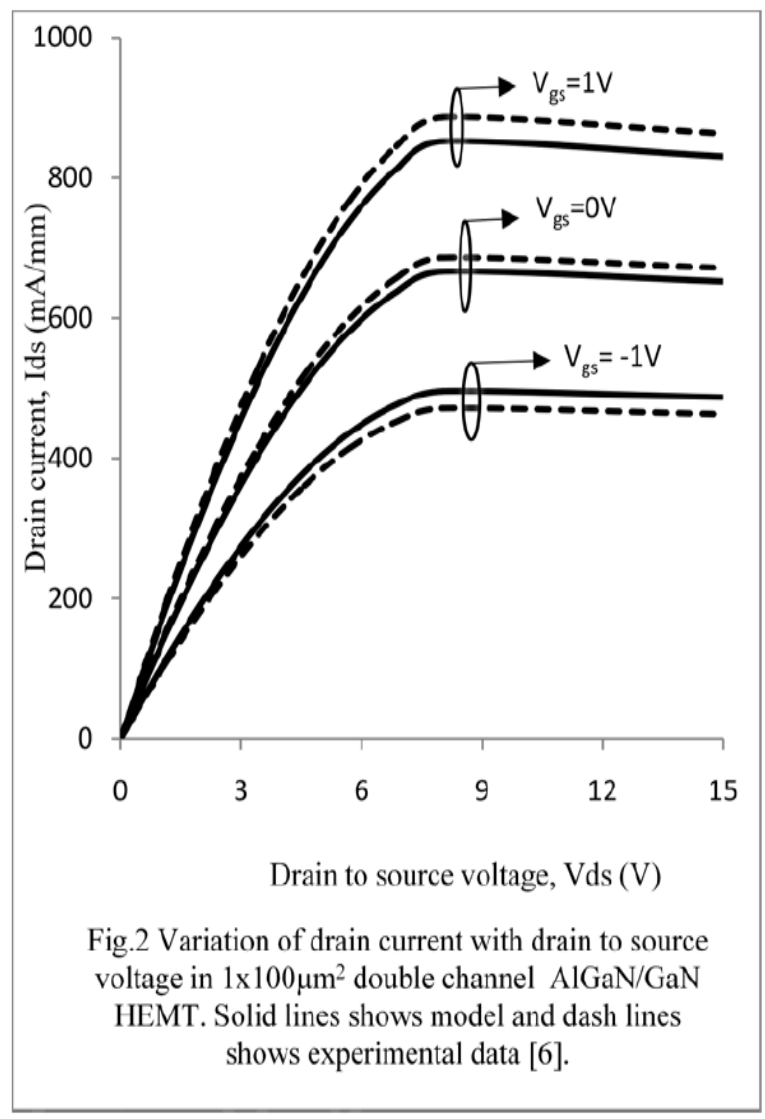

Fig.3 gives relationship between variation of drain current wih gate to source voltage at $\mathrm{V}_{\mathrm{ds}}=10 \mathrm{~V}$ in $1 \mathrm{x} 100 \mu \mathrm{m}^{2}$ gate area device structure. This dual channel AlGaN/GaN MODFET exhibit high current density with respect to conventional single channel AlGaN/GaN HEMT and also shows good control of gate voltage on device drain current[18]. The plotted results shows good agreement between model and the experimental data [6]. Minor difference in values might be attributed to miute gate and 
substrate leakage currents that are generally more dominent when the drain voltage rises to be more than the applied gate bias.

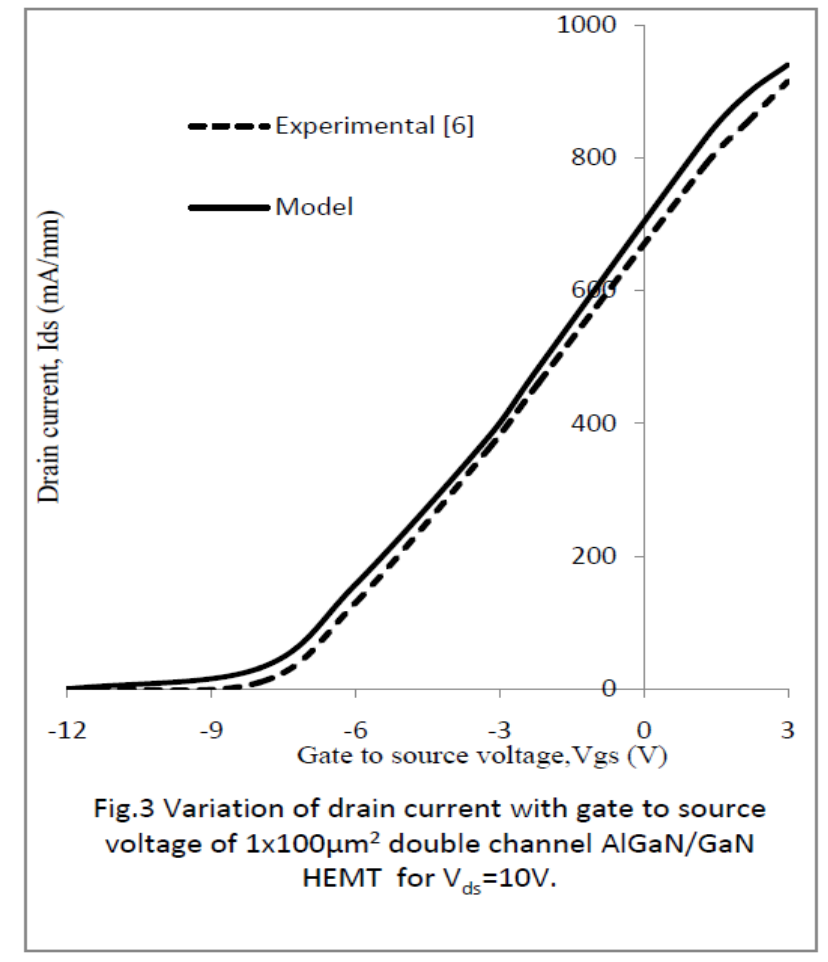

Fig.4 shows variation of transconductance with gate to souce voltage variation at $\mathrm{Vds}$ of $10 \mathrm{~V}$. There are two peaks in this curve proving double channel effects in derived model of dual channel MODFET. The peak value of transconductance, occurs near the gate bias at which 2-DEG charge density reaches the equilibrium value is $140 \mathrm{mS} / \mathrm{mm}$ at the gate voltage of $-1.6 \mathrm{~V}$. High transconductance in dual channel MODEFET is due to better charge control and improved carrier transport in the dual channel HEMTs. The reduction in value of transconductance at upper $\mathrm{V}_{\mathrm{gs}}$ values, is a characteristic feature and occurs becaue when 2-DEG sheet charge density approaching the equilibrium value, the current density becomes constant with the rise in gate to source voltage. Our model double hump characteristics in transconductence curve has been compared as reported by Rongming et. al. [6], which corresponds to the effective gate control on the upper and lower channels respectively. The results obtained from our proposed model are in close agreement with the published experimental data.

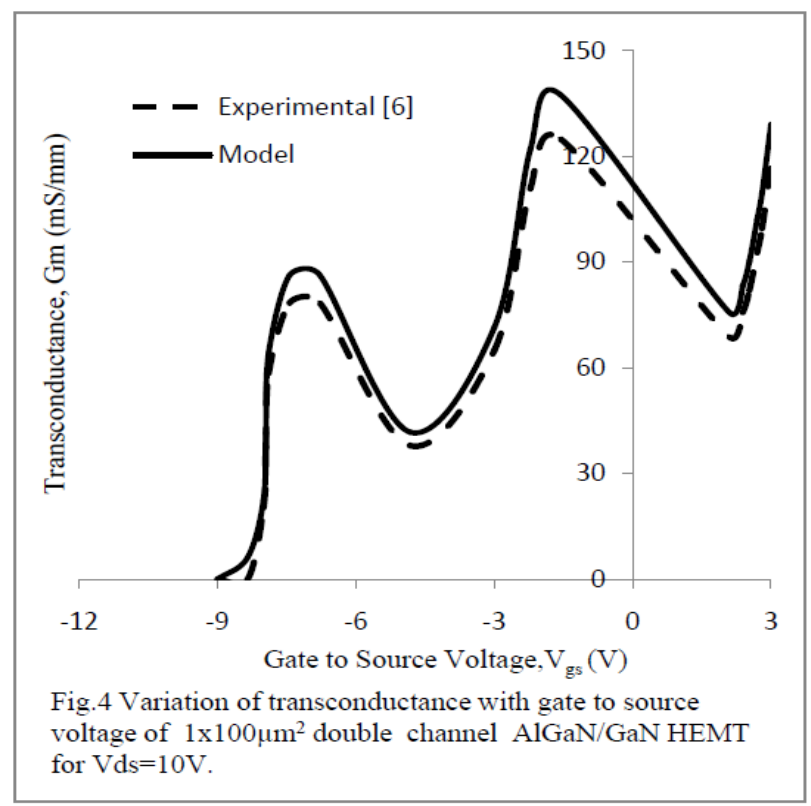

\section{CONCLUSION}

The 2-D model of current voltage characteristics and transconductance of dual channel AlGaN/GaN MODFET has been first time analysed by developing solid state physics based analytical model which incorporate pizoelectric and spontaneous polarization effects on $\mathrm{GaN}$ based device structures. The expressions for both the top and the bottom channel currents, have been obtained, by considering trapping/detrapping of surface states thus ensuring accuracy of our proposed model. The superiority of dual channel $\mathrm{Al}_{\mathrm{m}}$ $\mathrm{Ga}_{1-\mathrm{m}} \mathrm{N} / \mathrm{GaN}$ MODFETs in terms of overall current drive capability and supression of effect of current collapse in the device has been exmined. It is revealed that dual channel AlGaN/GaN MODFET has higher transconductance, better current drive capability and lesser current collapse than base line single channel AlGaN/GaN MODFETs. The results obtained from the developed model agree well with the published experimental results within the reasonable range of $\pm 5 \%$ thus proving accuracy of our proposed model.

From the above results it can be safely concluded that dual channel AlGaN/GaN MODFETs can be used to design high power microwave amplifiers, high frequency oscilators and modern radar and computer systems.

\section{REFERENCES}

[1]. O. Ambacher, J. Smart, J. R. Shealy, N. G. Weimann, K. Chu, M.Murphy, W. J. Schaff, L. F. Eastman, R. Dimitrov, L. Wittmer, M. Stutzmann, W. Rieger, and J. Hilsenbeck, "Two-dimensional electron gases induced by spontaneous and piezoelectric polarization charges in $\mathrm{N}$ - and Ga-face AlGaN/GaN heterostructures", J. Appl. Phys, vol. 85, no. 6, pp.3222-3233,Mar. 1999.

[2]. U. K. Mishra, P. Parikh, and Y. F. Wu, “AlGaN/GaN HEMTs-An Overview of Device Operation and Applications," Proc. IEEE, vol. 90, no.6, pp. 1022-1031, Jun 2002. 
[3]. K.Kasahara, N. Miyamoto, Y. Ando, Y. Okamoto, T. Nakayama, and M.Kuzuhara, "Ka-band $2.3 \mathrm{~W}$ power AlGaN/GaN hetrojunction FET," in IEDM Tech. Dig., pp. 667680, Dec. 2002.

[4]. W. Long, H. Ou, J. M. Kuo and K.K Chin., "DualMaterial Gate (DMG) Field Effect Transistor", IEEE Trans. Electron Devices, vol. 46, pp. 865-870, May 1999. [5]. W. Long et.al. "Methods for fabricating a dual material gate of a short channel field effect transistor," US Patent, US006153534, Nov 2000.

[6]. Roanming Chu, Yugang Zhou, Zie Liu, Deliang Wang, Kevin J.Chen, "AlGaN/GaN Double Channel HEMT," IEEE Transaction on Electron Devices, vol.52,no.4, Apr 2005.

[7]. P.M. Soloman and H Morkov modulation doped GaAs/AlGa As hetrojunction field effect transistor (MODFETs), ultrahighspeed device for supercomputers, IEEE Trans Electron Devices ED-31 (1984),1015-1027.

[8]. H. Morkov, H. Unlu and G.Ji, Principle of technology of MODFETs. Wiley, New York, 1991.

[9]. D. Delagebeaudeuf, NuyenT.Linh, "Metal-(n)AlGaAs/ GaAs Two Dimensional Electron Gas FET," IEEE Trans Electron Devices, vol.ED-29, no.6, pp.955-960, Jun.1982.

[10]. S.M. Zee,Physics of semiconductor devices, Wiley New York, $2^{\text {nd }} E d, 1981$.

[11]. E.S. Hellman, "The polarity of GaN: A critical review", MRS Internet J. Nitride Semiconduct. Res. 3, vol. 11, pp. 1$11,1998$.

[12]. Y.Zhang,I.P.Smorchkova, C.R.Elsass, S.Kellar, J.P.EIbbetson, S.Denbaars, U.K.Mishra ans J.Singh “” Charge control and mobility in AlGaN/ GaN Transistor:Experimental and theoretical studies" J.Appl, vol.87, no11, pp.7981-7987, 2000.

[13]. T.Li,R.P.Joshi and C.Fazi "Montecarlo evaluation of degeneracy and interface roughness effects on electron transport in AlGaN/ GaN hetrostructures" J.Appl,Phys vol.88, no. 2 , pp.829-837,2000

[14]. O. Ambacher, B.Foutz J. Smart, J. R. Shealy, N. G. Weimann, K. Chu, M.Murphy, A.J. Sierakowski, W. J. Schaff, L. F. Eastman, R. Dimitrov, M. Stutzmann and A Mitchell "Two-dimensional electron gases induced by spontaneous and piezoelectric polarization in undoped and doped AlGaN/GaN heterostructures", J. Appl. Phys, vol. 87, no. 1, pp.334-344, 2000.

[15]. Rashmi, A.Agrawal, S.Sen, S.Halder, R.S.Gupta, "Analytical model for DC characteristics and small signal parameters of $\mathrm{AlGaN} / \mathrm{GaN}$ Modulation Doped Field Effect Transistor for microwave applications", Microwave and Optical Technology Lett, vol.27, pp. 413-418, 2000.

[16]. P.P.Ruden, J.D.Albrcht, A.Sustandi, S.C.Binari, K.Ikkossi-Anastasiou, M.G.Ancona, R.L.Henery, D.D.koleske and A.E.Wikenden," Extrinsic performance limitations of $\mathrm{AlGaN} / \mathrm{GaN}$ heterostructure field effect transistor," MRS Internet, J Nitride Semiconduct. Res., 4S1, G6.35, 1999.

[17]. Rashmi, A. Kranti, S.Haldar, R.S.Gupta, "An accurate charge control model for spontaneous and piezoelectric polarization dependent two dimensional electron gas (2DEG) sheet charge density of lattice mismatched AlGaN/GaN HEMTs" Solid State Electron, vol. 46, no. 5, pp. 621-630, 2002.
[18]. Y.F.Wu, B.P.Kellar, P.Fini, S.Kellar, T.J.Jenkins, L.T.Kehias, S.P.Denbaares and U.K.Mishra, "Bias dependent microwave performance of $\mathrm{AlGaN} / \mathrm{GaN}$ MODFETs up to 100V," IEEE Electron Device Lett.,vol.18, no.6, pp.290-292, Jun1997.

[19]. Rahis Kumar Yadav, Pankaj Pathak, R M Mehra, "Analytical Modeling of Potential and Electric Field Distribution and Simulation of Large Signal Parameters for Dual Channel Dual Material Gate AlGaN/GaN High Electron Mobility Transistor." International Journal of Research and Scientific Innovation, vol.2, issue.5, pp.49-55, May 2015.

[20]. Rahis Kumar Yadav, Pankaj Pathak, R M Mehra, "Analytical DC Model of Double Channel Dual Material Gate $\mathrm{Al}_{\mathrm{m}} \mathrm{Ga}_{1-\mathrm{m}} \mathrm{N} / \mathrm{GaN}$ High Electron Mobility Transistor, Ultra High Speed Device for Microwave and Radar Applications", American International Journal of Research in Science, Technology, Engineering \& Mathematics, vol.3, issue.10, pp.334-341, March-May 2015

[21] Rahis Kumar Yadav, Pankaj Pathak, R M Mehra, "Current Voltage and Transconductance 2-D Model for Dual Material Gate $\mathrm{Al}_{\mathrm{m}} \mathrm{Ga}_{1-\mathrm{m}} \mathrm{N} / \mathrm{GaN}$ Modulation Doped Field Effect Transistor for High Frequency Microwave Circuit Applications", International Journal of Engineering Technology, Management and Applied Sciences, Vol.3, issue.6, pp.141-148, Jun 2015. 